\title{
Gamma and Beta Model of Growing and Rotating Planck Ball
}

\author{
U. V. S. Seshavatharam ${ }^{1}$ \\ Honorary Faculty, I-SERVE, S. No-42, Hitech City \\ Hyderabad-84, Telangana, India \\ Email: seshavatharam.uvs@gmail.com \\ S. Lakshminarayana ${ }^{2}$ \\ Department of Nuclear Physics, Andhra University \\ Visakhapatnam-03, AP, India \\ Email:Insrirama@gmail.com
}

\begin{abstract}
With reference to Planck scale, Mach's relation, increasing support for large scale cosmic anisotropy \& preferred directions and by introducing two new parameters Gamma and Beta, right from the beginning of Planck scale, we make an attempt to estimate ordinary matter density ratio, dark matter density ratio, mass, radius, temperature, age and expansion velocity (from and about the Planck mass in all directions). By considering $H_{0} \cong 70 \mathrm{~km} / \mathrm{sec} / \mathrm{Mpc}$, estimated current cosmic mass, radius, total matter density, expansion velocity, temperature and age are: $4.3352 \times 10^{53} \mathrm{~kg}$, $3.207 \times 10^{26} \mathrm{~m}, 3.138 \times 10^{-27} \mathrm{~kg} \cdot \mathrm{m}^{-3}, 2.43 \mathrm{c}, \quad 2.721 \mathrm{~K}$ and 19.78 Billion years respectively. Point to be noted is that, with reference to Planck scale, ratio of Hubble parameter $H_{t}$ to angular velocity $\omega_{t}$ can be expressed with $\left(H_{t} / \omega_{t}\right) \cong \gamma_{t} \cong\left[1+\ln \left(H_{p l} / H_{t}\right)\right] \cong \sqrt{3 H_{t}^{2} c^{2} / 8 \pi G\left(a T_{t}^{4}\right)}$ where $H_{p l}$ represents Planck scale angular velocity and $\left(a T_{t}^{4}\right)$ is the thermal energy density. $\left(H_{0} / \omega_{0}\right) \cong \gamma_{0} \cong 141.26$ and $\omega_{0} \cong 1.606 \times 10^{-20} \mathrm{rad} / \mathrm{sec} \cong 5.068 \times 10^{-13} \mathrm{rad} / \mathrm{year}$. It needs further study. Proceeding further, from the beginning of Planck scale, a) With a 'decreasing' trend of total matter density ratio, cosmic expansion velocity can be shown to be increasing. b) With an 'increasing' trend of total matter density ratio, cosmic expansion velocity can be shown to be decreasing. c) With a constant trend of total matter density ratio, cosmic expansion velocity can be shown to be constant. In this model, in understanding the currently believed cosmic acceleration, there is no need to consider dark energy.
\end{abstract}

Keywords: Planck scale; Mach's relation; quantum cosmology; critical density; ordinary matter; dark matter; expansion velocity; angular velocity; Hubble's law;

PACS Nos: 04.20.-q, 04.60.-m, 04.50.Kd, , 98.80.Qc, 98.80.Bp, 98.80.Es

\section{Introduction}

According to current notion of modern cosmology, if the known laws of physics are extrapolated to the highest density regime, the result is a singularity which is typically associated with the Big Bang[1,2]. Even though it is intriguing to consider 'big bang' hypothesis, physics associated with big bang notion is speculative, confusing and misleading with respect to 'Planck scale' physics. Important demerits of big bang notion can be understood with the following points:

1) Preconditions of big bang are unclear and unknown;

2) No quantitative description is available for the matter content associated with the big bang event;

3) Physical reasons that led to big bang are unclear and unknown;

4) Quantitative description for big bang bursting force or pressure is unclear and unknown; 
5) Whether big bang followed known physical laws are not - is also unclear and unknown;

6) Quantum information associated with big bang is unclear and unknown;

7) Within a fraction of second, how, big bang allowed 'inflation' to happen? - is a puzzling issue;

8) Applying Planck scale physics to big bang notion is a confusing issue;

9) Whether pre big bang or post big bang constitutes dark matter - is unclear and unknown;

10) Role of dark energy in big bang - is another complicated and questionable issue;

If one is willing to think in this way, it may not be wrong to say that, 'big bang' is a 'meta' physical concept and an ad hoc physical event. At any stage of its development and testing, any theoretical model footing on a metaphysical concept, will certainly leads to ambiguity and uncertainty. In this critical scenario, in a quantitative approach, it may not be wrong to consider a 'growing' or 'evolving' phase of 'Planck scale'. Even though massive nature is unclear - with known physical laws, Planck scale can be assigned with certain 'mass', certain 'radius', certain 'volume', certain 'density', certain 'temperature' and certain 'pressure' etc. Clearly speaking, Planck mass can be considered as a characteristic massive seed of the evolving universe and big bang can be replaced with an evolving Planck ball. Planck mass can be called as the 'baby universe' or 'baby Planck ball'.

Since nothing is known, it is absolutely not possible to simulate a big bang, but with future science, engineering and technology, it is certainly possible to simulate any 'Planck scale' physical event. Till that time, cosmic observations can be analyzed with a notion of 'growing Planck ball'. Thus, by replacing 'big bang' [3,4] with a growing Planck ball and considering 'Mach's relation' [7-9] as a deep cosmic probe, in a hypothetical approach, an evolving model of quantum cosmology can be developed [10-12]. Since Planck scale is associated with Quantum theory and 'spin' is a basic property of quantum mechanics, it may not be wrong to consider a growing and rotating model of a Planck ball.

To make the model sensible and practical, in a quantitative approach, we assume that, ratio of Hubble parameter to angular velocity is, $\left(H_{t} / \omega_{t}\right) \cong \gamma_{t} \cong\left[1+\ln \left(H_{p l} / H_{t}\right)\right] \cong \sqrt{3 H_{t}^{2} c^{2} / 8 \pi G\left(a T_{t}^{4}\right)}$ where $\left(H_{p l}, H_{t}\right)$ represent Planck scale and time dependent Hubble parameters respectively and $\left(a T_{t}^{4}\right)$ is the thermal energy density. Considering $\gamma_{0}$, current cosmic temperature and current Hubble parameter can be fitted. Right from the beginning of Planck scale: 1) Considering $G M_{t} \cong R_{t} c^{2}$ and $\left(3 H_{t}^{2} c^{2} / 8 \pi G\right)$, an attempt is made to develop simple relations for estimating cosmic mass, radius and expansion velocity (referred to 'from and about' the baby universe in all directions). 2) Defining $\beta_{t} \cong\left(1+\sqrt{\gamma_{t}}\right) / 2$, another attempt is made to develop 'model' relations for estimating dark matter density ratio and ordinary matter density ratio. Thus, in analogy with current 'cosmic acceleration', using $\left(\gamma_{t}, \beta_{t}\right)$, we proposed simple relations for a decreasing trend of total matter density ratio with increasing trend of cosmic expansion velocity.

\subsection{Motivating points}

1) Mach's principle [13] is one of the iconic principles underlying general theory of relativity and can be given a priority in developing a workable or unified model of cosmology.

2) Cosmic expansion, Lambda term, dark matter, cosmic temperature, inflation, cosmic acceleration and dark energy and vacuum energy are different concepts, by using which alternative models of GTR are emerging and are being extended in many ways. In this sequence, quantum cosmology can also be given some consideration.

3) Quantum cosmology is a wide range physical model intended for understanding the in-built cosmological quantum phenomena on small scale as well as large scale distances. So far, progress 
in this direction is very nominal and 'GTR' needs a serious review with reference to 'quantum cosmology'.

4) When universe is able to give birth to atoms, elementary parrticles and photons that show quantrum behaviour, universe can certainly be considered as a quantum gravitational object for ever.

5) What to quantize? How to quantize? When to quantize? and What to measure? etc. are some interesting questions in current quantum cosmology and need a special focus. In this context, cosmic temperature can be considered as a characteristic feature of quantum cosmology.

6) With reference to particle physics, current technological limits on particle colliding energy, unidentified/unseen particles, unknown particle interactions and incomplete final unification scheme - to some extent, one can hopefully believe in the existence of dark matter [14].

7) Basically, 'dark energy' was proposed for understanding cosmic acceleration. Careful analysis of improved supernovae data suggets that universe is coasting at constant veleocity and evidence for acceleration is only marginal [15-17]. In this context, now a days, a great debate has been initiated among mainstream cosmologists on the existence of dark energy [18-21]. According to a recent study [22], the nature of dark energy is 'dynamic' and conceptually seems to deviate from the famous cosmological constant or vaccum energy. According to another new study [23], evidence for dynamical dark energy is very poor.

8) Density perturbations [21] and interaction between dark mtter and baryons [20] seem to play a crucial role in understanding observed cosmic acceleration and need of introducing dark energy seems to be ad-hoc.

9) Even though redshift is an index of cosmic expansion, without knowing the actual galactic distances and actual galactic receding speeds, with $100 \%$ confidence level, it may not be possible to decide the absolute nature of cosmic expansion rate.

10) If the Universe is the same in all directions, as the big bang models require, the hot spots and cold spots of CMBR in the afterglow of the big bang should be randomly splattered about the sky - the big temperature splotches and the small temperature goose pimples should have no preferred direction. The fact that they are aligned along the axis of evil leads Kate Land and Joao Magueijo [24] to suggest that, may be the assumptions behind the big bang models are wrong. In other words, the Universe is not the same in all places or directions, but has a special direction.

11) Considering a sample of 355 optically polarized quasars with accurate linear polarization measurements, Hutsem'ekers et al $[25,26]$, demonstrated that quasar polarization angles are definitely not randomly oriented over the sky. Polarization vectors appear coherently oriented over very large spatial scales, in regions located at both low and high redshifts and characterized by different preferred directions. These characteristics make the alignment effect difficult to explain in terms of local mechanisms, namely a contamination by interstellar polarization in our Galaxy.

12) According to Shamik Ghosh et al [27] -The tantalizing possibility that the cosmological principle may be violated is indicated by many observations. The most prominent of these effects is the socalled Virgo alignment, which refers to a wide range of phenomena indicating a preferred direction pointing towards Virgo. The Square Kilometer Array has the capability to convincingly test several of these effects. These include the dipole anisotropy in radio polarization angles [28], the dipole in the number counts and sky brightness [29-33] and in the polarized number counts and polarized flux [34]. These observations may indicate that we need to go beyond the standard Big Bang cosmology. Alternatively they may be explained by preinflationary anisotropic and/or inhomogeneous modes $[35,36]$. In either case, confirmation of this alignment effect is likely to revolutionize cosmology.

13) According to Wen Zhao and Larissa Santos [37] - The foundation of modern cosmology relies on the so-called cosmological principle which states a homogeneous and isotropic distribution of matter in the universe on large scales. However, recent observations, such as the temperature anisotropy of the cosmic microwave background (CMB) radiation, the motion of galaxies in the 
universe, the polarization of quasars and the acceleration of the cosmic expansion, indicate preferred directions in the sky. If these directions have a cosmological origin, the cosmological principle would be violated, and modern cosmology should be reconsidered.

14) Nature loves symmetry. Subject of cosmic 'rotation' is not new and not against to General theory of relativity [38-43]. Quantum mechanics point of view, 'spin' is a basic and characteristic property. Quantum gravity point of view, it is reasonable to review the currently believed 'standard cosmology' with reference to cosmic rotation. In this context, in literature one can find interesting articles on cosmic rotation and angular velocity [44-60].

\section{Assumptions, concepts and relations}

\subsection{Nomenclatures}

1) $\quad\left(\Omega_{O M}\right)_{t}=$ Ratio of ordinary matter density to critical density.

2) $\quad\left(\Omega_{D M}\right)_{t}=$ Ratio of dark matter density to critical density.

3) $H_{t}=$ Hubble parameter and $H_{p l}=$ Planck scale Hubble parameter.

4) $\omega_{t}=$ Cosmic angular velocity and $\omega_{p l}=$ Planck scale angular velocity.

5) $\quad\left(V_{\text {exp }}\right)_{t}=$ Cosmic expansion velocity from and about the baby universe or baby Planck ball.

6) $\quad\left(M_{O M}\right)_{t}=$ Cosmic ordinary mass content, $\left(M_{D M}\right)_{t}=$ Cosmic dark matter content.

7) $\quad\left(M_{O M}+M_{D M}\right)_{t} \cong M_{t}=$ Total matter content $=$ Total mass of evolving Planck ball.

8) $\quad R_{t}=$ Cosmic radius associated with $M_{t}=$ Radius of evolving Planck ball.

9) $T_{t}=$ Cosmic temperature.

10) $\gamma_{t} \cong\left(\frac{H_{t}}{\omega_{t}}\right) \cong\left[1+\ln \left(\frac{H_{p l}}{H_{t}}\right)\right] \cong \sqrt{\frac{3 H_{t}^{2} c^{2}}{8 \pi G\left(a T_{t}^{4}\right)}}=$ Ratio of Hubble parameter to angular velocity.

11) $\beta_{t} \cong \frac{1+\sqrt{\gamma_{t}}}{2}=$ A new number defined to be associated with ordinary matter density ratio and dark matter density.

12) $\left(d_{g}\right)_{t}=$ Galactic distance from and about the baby universe or baby Planck ball.

13) $\left(v_{g}\right)_{t}=$ Galactic receding speed from and about the baby universe or baby Planck ball.

Note-1: For the above symbols, subscript 0 denotes current value and subscript $p l$ denotes Planck scale value.

\subsection{Proposed assumptions}

With respect to our earlier publications [61-65], in this paper we review the basic assumptions.

1) Planck scale and Mach's relation play a crucial role in entire cosmic evolution.

2) Ratio of Hubble parameter to angular velocity is $\gamma_{t} \cong\left(\frac{H_{t}}{\omega_{t}}\right) \cong\left[1+\ln \left(\frac{H_{p l}}{H_{t}}\right)\right] \cong \sqrt{\frac{3 H_{t}^{2} c^{2}}{8 \pi G\left(a T_{t}^{4}\right)}}$.

3) Ordinary matter and dark matter, both, play a crucial role in estimating cosmic expansion velocity. 
Note-2: Based on these assumptions, from the beginning of Planck scale, a) With a 'decreasing' trend of total matter density ratio, cosmic expansion velocity can be shown to be increasing. b) With an 'increasing' trend of total matter density ratio, cosmic expansion velocity can be shown to be decreasing. c) With a constant trend of total matter density ratio, cosmic expansion velocity can be shown to be constant.

\subsection{Choosing the magnitude of $H_{0}$}

1) As per the 2015 Planck data [66]: $H_{0} \cong(67.31 \pm 0.96) \mathrm{km} / \mathrm{sec} / \mathrm{Mpc}$ and the present temperature of the $\mathrm{CMB}$ radiation is, $T_{0} \cong(2.722 \pm 0.027) \mathrm{K}$.

2) According to the advanced observational data analysis by Riess et al [67] current best value of $H_{0} \cong(73.24 \pm 1.74) \mathrm{km} / \mathrm{sec} / \mathrm{Mpc}$.

3) With reference to $T_{0} \cong 2.722 \mathrm{~K}$ and our proposed set of concepts, in this paper, we choose, $H_{0} \cong 70 \mathrm{~km} / \mathrm{sec} / \mathrm{Mpc} \cong 2.26853 \times 10^{-18} \sec ^{-1}$. This value seems to lie in between (67.31 and 73.24) $\mathrm{km} / \mathrm{sec} / \mathrm{Mpc}$.

\subsection{The Planck scale in entire cosmic evolution}

So far no mainstream cosmological model implemented Planck scale in current cosmic evolution. In this complicated situation, in a positive approach, we make an attempt to implement the 'Planck scale' in the entire cosmic evolution. With further study, our approach can be developed for a better understanding. Based on quantum gravity, we define the Planck scale Hubble parameter, $H_{p l} \cong \sqrt{\frac{c^{5}}{G \hbar}} \cong 1.854921 \times 10^{43} \mathrm{sec}^{-1}$. To proceed further, we define that,

$$
\begin{gathered}
\left(\frac{H_{t}}{\omega_{t}}\right) \cong \gamma_{t} \cong\left[1+\ln \left(\frac{H_{p l}}{H_{t}}\right)\right] \cong \sqrt{\left(\frac{3 H_{t}^{2} c^{2}}{8 \pi G\left(a T_{t}^{4}\right)}\right)} \\
\frac{3 \omega_{t}^{2} c^{2}}{8 \pi G} \cong a T_{t}^{4} \quad \text { and } \quad \omega_{\mathrm{t}} \cong \sqrt{\frac{8 \pi G a T_{t}^{4}}{3 c^{2}}}
\end{gathered}
$$

Based on this relation, if defined $H_{p l} \cong 1.854921 \times 10^{43} \sec ^{-1}$, one can choose different values of $\gamma$ in between $\gamma_{p l} \cong 1$ and $\gamma_{0} \cong 141.2564$. For each assumed value of $H$, one can get a corresponding $\gamma$ and all other physical parameters can be estimated. For the Planck scale, $\sqrt{\left(\frac{3 H_{p l}^{2} c^{2}}{8 \pi G\left(a T_{p l}^{4}\right)}\right)} \cong \gamma_{p l} \cong 1$ and for the current case, $\sqrt{\left(\frac{3 H_{0}^{2} c^{2}}{8 \pi G\left(a T_{0}^{4}\right)}\right)} \cong \gamma_{0} \cong\left[1+\ln \left(\frac{H_{p l}}{H_{0}}\right)\right] \cong\left(\frac{H_{0}}{\omega_{0}}\right) \cong 141.2564$. In a simplified form, cosmic temperature can be expressed as,

$$
T_{t} \cong \frac{1}{\sqrt{\gamma_{t}}}\left(\frac{3 H_{t}^{2} c^{2}}{8 \pi G a}\right)^{\frac{1}{4}} \cong \frac{1}{\sqrt{\gamma_{t}}}\left\{\frac{0.652632 \hbar \sqrt{H_{p l} H_{t}}}{k_{B}}\right\}
$$

If one is willing to define, critical temperature as $\left(T_{c}\right)_{t} \cong\left(\frac{3 H_{t}^{2} c^{2}}{8 \pi G a}\right)^{\frac{1}{4}}$, then, 


$$
\sqrt{\gamma_{t}} \cong \frac{\text { Critical temperature }}{\text { Actual temperature }} \cong \frac{\left(T_{c}\right)_{t}}{T_{t}}
$$

\section{To estimate the cosmic mass, radius and expansion velocity}

Let,

$$
\begin{array}{r}
\left(M_{O M}+M_{D M}\right)_{t} \cong M_{t} \cong\left[\left(\Omega_{O M}\right)_{t}+\left(\Omega_{D M}\right)_{t}\right]\left(\frac{3 H_{t}^{2}}{8 \pi G}\right)\left(\frac{4 \pi}{3} R_{t}^{3}\right) \\
G\left(M_{O M}+M_{D M}\right)_{t} \cong G M_{t} \cong R_{t} c^{2} \\
R_{t} \cong \frac{G\left(M_{O M}+M_{D M}\right)_{t}}{c^{2}} \cong \frac{G M_{t}}{c^{2}}
\end{array}
$$

Based on these relations, it is possible to show that,

$$
\begin{gathered}
R_{t} \cong \frac{G\left(M_{O M}+M_{D M}\right)_{t}}{c^{2}} \cong \sqrt{\frac{2}{\left(\Omega_{O M}+\Omega_{D M}\right)_{t}}}\left(\frac{c}{H_{t}}\right) \\
\left(V_{\text {exp }}\right)_{t} \cong R_{t} H_{t} \cong \sqrt{\frac{2}{\left(\Omega_{O M}+\Omega_{D M}\right)_{t}}}(c) \\
\left(M_{O M}+M_{D M}\right)_{t} \cong M_{t} \cong \sqrt{\frac{2}{\left(\Omega_{O M}+\Omega_{D M}\right)_{t}}}\left(\frac{c^{3}}{G H_{t}}\right) \cong\left(\frac{c^{2}\left(V_{\text {exp }}\right)_{t}}{G H_{t}}\right)
\end{gathered}
$$

Based on relations (6 to 10$)$ and in terms of $\left(\Omega_{O M}\right)_{t}$ and $\left(\Omega_{D M}\right)_{t}$,

$$
\begin{gathered}
\left(M_{O M}\right)_{t} \cong\left[\frac{\left(\Omega_{O M}\right)_{t}}{\left(\Omega_{O M}+\Omega_{D M}\right)_{t}}\right]\left(\frac{c^{2}\left(V_{\text {exp }}\right)_{t}}{G H_{t}}\right) \\
\left(M_{D M}\right)_{t} \cong\left[\frac{\left(\Omega_{D M}\right)_{t}}{\left(\Omega_{O M}+\Omega_{D M}\right)_{t}}\right]\left(\frac{c^{2}\left(V_{\text {exp }}\right)_{t}}{G H_{t}}\right)
\end{gathered}
$$

\section{Understanding the trend of ordinary and dark matter density ratios}

With the help of defined $\gamma_{t} \cong\left(\frac{H_{t}}{\omega_{t}}\right) \cong\left[1+\ln \left(\frac{H_{p l}}{H_{t}}\right)\right]$, on ad-hoc basis and with reference to the current observed values of $\left(\Omega_{O M}\right)_{0}$ and $\left(\Omega_{D M}\right)_{0}$, we are making an attempt to estimate the past values of $\left(\Omega_{O M}\right)_{t}$ and $\left(\Omega_{D M}\right)_{t}$. In this context, the basic question to be answered is: Is there any scope for the existence for dark matter at Planck scale? It needs further study. In a positive approach, we hope that there exits ordinary matter as well dark matter at Planck scale in certain proportions.

\subsection{Current ordinary matter density ratio}

If it is assumed that, 


$$
\left\{\left[\left(M_{O M}\right)_{0} c^{2}\right] /\left[\frac{4 \pi}{3} R_{0}^{3}\right]\right\} \propto \sqrt{\left(\frac{3 H_{0}^{2} c^{2}}{8 \pi G}\right)\left(a T_{0}^{4}\right)}
$$

As per relation (1),

$$
\left\{\left[\left(M_{O M}\right)_{0} c^{2}\right] /\left[\frac{4 \pi}{3} R_{0}^{3}\right]\right\} \propto \frac{1}{\gamma_{0}}\left(\frac{3 H_{0}^{2} c^{2}}{8 \pi G}\right)
$$

By considering an ad-hoc proportionality coefficient of the form, $\beta_{0} \cong\left(\frac{1+\sqrt{\gamma_{0}}}{2}\right)$,

$$
\begin{aligned}
& \left\{\left[\left(M_{\text {OM }}\right)_{0} c^{2}\right] /\left[\frac{4 \pi}{3} R_{0}^{3}\right]\right\} \cong \frac{\beta_{0}}{\gamma_{0}}\left(\frac{3 H_{0}^{2} c^{2}}{8 \pi G}\right) \\
& \text { and }\left(\Omega_{O M}\right)_{0} \cong\left(\frac{1}{\gamma_{0}}\right)\left(\frac{1+\sqrt{\gamma_{0}}}{2}\right) \cong \frac{\beta_{0}}{\gamma_{0}} \cong 0.04561 \\
& \left\{\left[\left(M_{O M}\right)_{0} c^{2}\right] /\left[\frac{4 \pi}{3} R_{0}^{3}\right]\right\} \cong \beta_{0}\left(\frac{3 H_{0} \omega_{0} c^{2}}{8 \pi G}\right)
\end{aligned}
$$

\subsection{Current dark matter density ratio}

Like the case of ordinary matter density,

$$
\left\{\left[\left(M_{D M}\right)_{0} c^{2}\right] /\left[\frac{4 \pi}{3} R_{0}^{3}\right]\right\} \propto \sqrt{\left(\frac{3 H_{0}^{2} c^{2}}{8 \pi G}\right)\left(a T_{0}^{4}\right)}
$$

As per relation (1),

$$
\left\{\left[\left(M_{D M}\right)_{0} c^{2}\right] /\left[\frac{4 \pi}{3} R_{0}^{3}\right]\right\} \propto \frac{1}{\gamma_{0}}\left(\frac{3 H_{0}^{2} c^{2}}{8 \pi G}\right)
$$

By considering an ad-hoc proportionality coefficient of the form, $\beta_{0}^{2} \cong\left(\frac{1+\sqrt{\gamma_{0}}}{2}\right)^{2}$,

$$
\begin{aligned}
& \left\{\left[\left(M_{D M}\right)_{0} c^{2}\right] /\left[\frac{4 \pi}{3} R_{0}^{3}\right]\right\} \cong \frac{\beta_{0}^{2}}{\gamma_{0}}\left(\frac{3 H_{0}^{2} c^{2}}{8 \pi G}\right) \\
& \text { and }\left(\Omega_{D M}\right)_{0} \cong\left(\frac{1}{\gamma_{0}}\right)\left(\frac{1+\sqrt{\gamma_{0}}}{2}\right)^{2} \cong \frac{\beta_{0}^{2}}{\gamma_{0}} \cong 0.29384 \\
& \left\{\left[\left(M_{D M}\right)_{0} c^{2}\right] /\left[\frac{4 \pi}{3} R_{0}^{3}\right]\right\} \cong \beta_{0}^{2}\left(\frac{3 H_{0} \omega_{0} c^{2}}{8 \pi G}\right)
\end{aligned}
$$

\subsection{General expressions for understanding the trend of ordinary and dark matter density ratios}

By extrapolating the coefficient $\beta_{t}$ to past and future as,

$$
\beta_{t} \cong\left(\frac{1+\sqrt{\gamma_{t}}}{2}\right) \text { and } \beta_{t}^{2} \cong\left(\frac{1+\sqrt{\gamma_{t}}}{2}\right)^{2}
$$



A) $\left(\Omega_{O M}\right)_{t} \cong\left(\frac{1}{\gamma_{t}}\right)\left(\frac{1+\sqrt{\gamma_{t}}}{2}\right) \cong \frac{\beta_{t}}{\gamma_{t}}$
В) $\left(\Omega_{D M}\right)_{t} \cong\left(\frac{1}{\gamma_{t}}\right)\left(\frac{1+\sqrt{\gamma_{t}}}{2}\right)^{2} \cong \frac{\beta_{t}^{2}}{\gamma_{t}}$
C) $\frac{\left(\Omega_{D M}\right)_{t}}{\left(\Omega_{O M}\right)_{t}} \cong\left(\frac{1+\sqrt{\gamma_{t}}}{2}\right) \cong \beta_{t}$;
D) $\left(\Omega_{O M}+\Omega_{D M}\right)_{t} \cong \frac{\beta_{t}+\beta_{t}^{2}}{\gamma_{t}}$

See figure 1 plotted with relations (22A) and (22B). With reference to critical density, dashed blue curve represents the trend of ordinary matter density ratio and black curve represents the trend of dark matter density ratio. With reference to the 'status of existence' of ordinary matter and dark matter and with further research, various relations for $\beta_{t}$ can be developed by using which, in near future, trend of ordinary and dark matter density ratios can be understood right from the beginning of Planck scale.

Figure 1: Decreasing trend of ordinary and dark matter density ratios

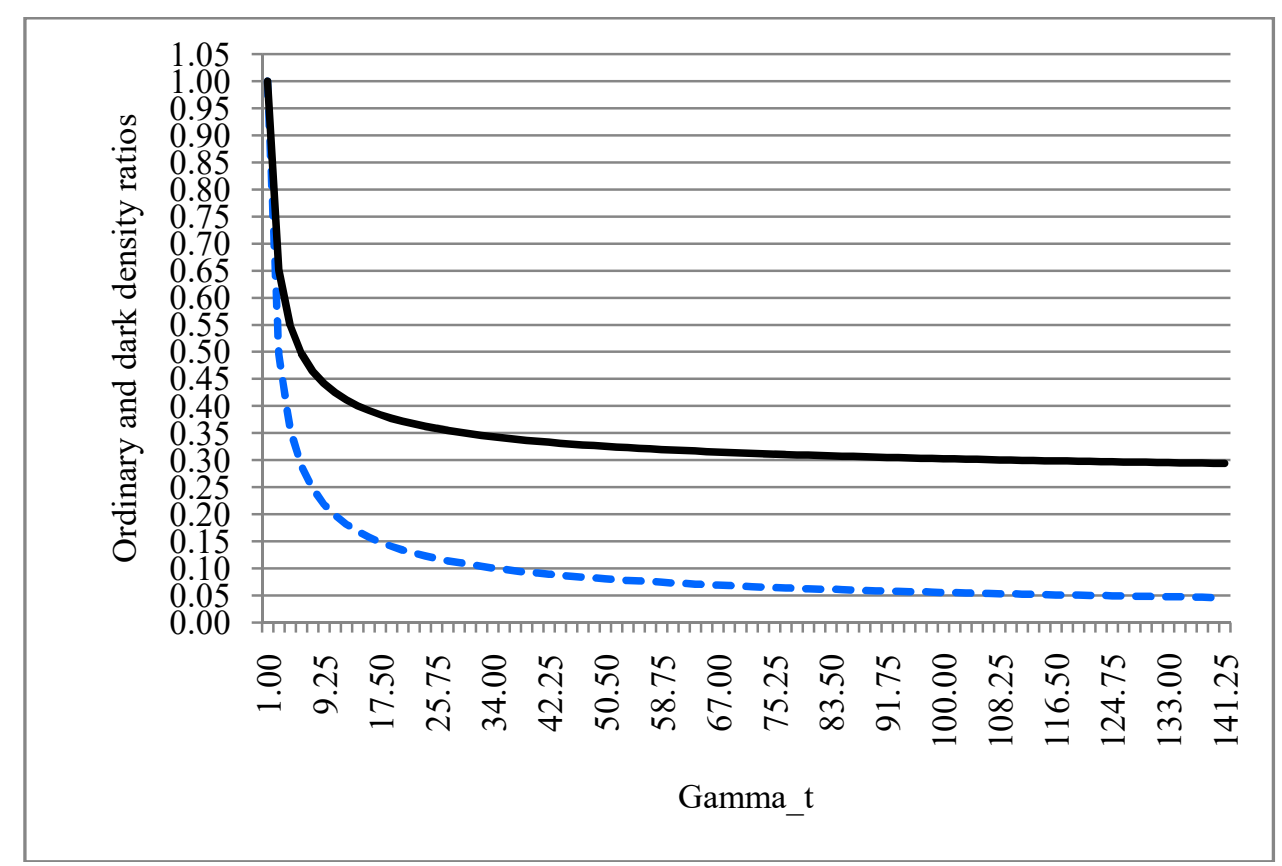

Note-4: If $\left(\Omega_{O M}+\Omega_{D M}\right)_{0} \cong\left(\beta_{0}^{2} / \gamma_{0}\right) \cong 0.29384$ and $\left(\Omega_{O M}\right)_{0} \cong\left(\beta_{0} / \gamma_{0}\right) \cong 0.04561$, then it is possible to show that, $\quad\left(\Omega_{D M}\right)_{0} \cong\left[\left(\beta_{0}^{2} / \gamma_{0}\right)-\left(\beta_{0} / \gamma_{0}\right)\right] \cong\left[\left(\beta_{0}^{2}-\beta_{0}\right) / \gamma_{0}\right] \cong 0.24823$. Extrapolating it to Planck's scale, $\left(\Omega_{D M}\right)_{p l} \cong\left[\left(\beta_{p l}^{2}-\beta_{p l}\right) / \gamma_{p l}\right] \cong 0$. It implies that, at planck scale, there exists no dark matter! During its evolution, as the Planck ball is growing, $\left(\Omega_{D M}\right)$ starts from 'zero' and reaches to a current state of 0.25 whereas $\left(\Omega_{O M}\right)$ starts from 1 and reaches to a current state of 0.0456 . It is for further study. See figure 2 (plotted with relation 22D) for a drcreasing trend of total matter density ratio. 
Figure 2: Decreasing trend of total matter density ratio

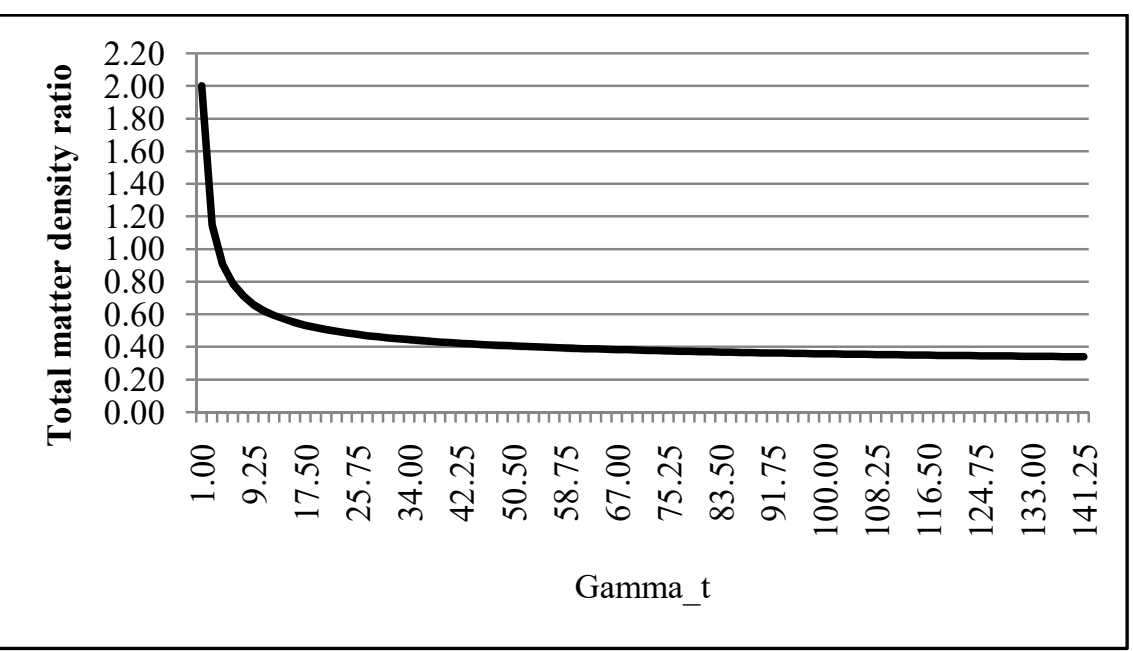

\section{Current and Planck scale physical parameters}

See table 1 for various cosmic physical parameters associated with current and Planck scales.

\begin{tabular}{|l|l|}
\hline \multicolumn{2}{|c|}{ Table 1: Current and Planck scale cosmic physical } \\
parameters
\end{tabular}




\begin{tabular}{|l|l|}
\hline$\left[\left(M_{O M}\right)_{0}+\left(M_{D M}\right)_{0}\right]$ & {$\left[\left(M_{O M}\right)_{p l}+\left(M_{D M}\right)_{p l}\right]$} \\
$\cong M_{0} \cong 4.3352 \times 10^{53} \mathrm{~kg}$ & $\cong M_{p l} \cong 2.176 \times 10^{-8} \mathrm{~kg}$ \\
\hline$t_{0} \cong \frac{2 R_{0}}{\left(\left(V_{\text {exp }}\right)_{0}+\left(V_{\text {exp }}\right)_{p l}\right)}$ & $t_{p l} \cong 0$ \\
$\cong 19.78$ BillionYears & \\
\hline
\end{tabular}

\section{Cosmic scale factor and red shift}

With reference to the proposed relations (1) and (3) and with reference to the current definitions of cosmic redshift and scale factor, it is possible to show that,

$$
\begin{aligned}
& \left(\frac{1}{a} \cong(z+1) \cong \frac{T_{t}}{T_{0}}\right) \cong \sqrt{\frac{\gamma_{0} H_{t}}{\gamma_{t} H_{0}}} \cong \sqrt{\frac{\gamma_{0}}{\gamma_{t}}} \sqrt{\frac{H_{t}}{H_{0}}} \\
& \cong \sqrt{\frac{\gamma_{0}}{\gamma_{t}}}\left\{\exp \left(\frac{\gamma_{0}-\gamma_{t}}{2}\right)\right\} \cong \sqrt{\left(\frac{\gamma_{0}}{\gamma_{t}}\right) \exp \left(\gamma_{0}-\gamma_{t}\right)}
\end{aligned}
$$

Redshift can be expressed in the following form.

$$
z \cong \sqrt{\left(\frac{\gamma_{0}}{\gamma_{t}}\right) \exp \left(\gamma_{0}-\gamma_{t}\right)}-1
$$

We are working on interpreting this relation and it needs further study.

\section{To estimate the current cosmic age}

From the beginning of cosmic evolution, based on the proposed cosmic expansion velocities, cosmic age can be approximated with the following relation.

$$
t \cong \frac{\left(R_{t}-R_{p l}\right)}{\left[\left(\left(V_{\exp }\right)_{t}+\left(V_{\exp }\right)_{p l}\right) / 2\right]}
$$

where $\left[\left(\left(V_{\exp }\right)_{t}+\left(V_{\exp }\right)_{p l}\right) / 2\right]$ can be considered as average expansion velocity.

For the current case,

$$
\begin{aligned}
t_{0} & \cong \frac{\left(R_{0}-R_{p l}\right)}{\left[\left(\left(V_{\exp }\right)_{0}+\left(V_{\text {exp }}\right)_{p l}\right) / 2\right]} \cong \frac{2 R_{0}}{\left(\left(V_{\text {exp }}\right)_{0}+\left(V_{\text {exp }}\right)_{p l}\right)} \\
& \cong 19.78 \text { BillionYears }
\end{aligned}
$$

For a temperature of $3000 \mathrm{~K}$, it is possible to show that, 


$$
\left.\begin{array}{l}
H_{3000 K} \cong 2.49 \times 10^{-12} \mathrm{sec}^{-1} \\
\gamma_{3000 K} \cong 1+\ln \left(\frac{H_{p l}}{H_{3000 K}}\right) \cong 127.34774 \\
Z_{3000 K} \cong \sqrt{\gamma_{3000 K}}-1 \cong 10.285 \\
z_{3000 K} \cong \sqrt{\frac{\gamma_{0}}{\gamma_{3000 K}} \exp \left(\gamma_{0}-\gamma_{3000 K}\right)}-1 \cong 1102.407
\end{array}\right\}
$$

Cosmic age corresponding to a temperature of $\mathrm{T}=3000 \mathrm{~K}$ can be estimated to be,

$$
t_{3000 K} \cong \frac{\left(R_{3000 K}-R_{p l}\right)}{\left[\left(\left(V_{\exp }\right)_{3000 K}+\left(V_{\text {exp }}\right)_{p l}\right) / 2\right]} \cong 17987.07 \text { Years }
$$

This estimation is 21.13 times less than the current estimations and needs further study.

\section{Velocity and distance relation}

In all directions, from and about the hypothetical baby Planck ball, current galactic receding speeds can be approximated with,

$$
\left(v_{g}\right)_{0} \cong\left(\frac{\left(d_{g}\right)_{0}}{R_{0}}\right)\left(V_{\exp }\right)_{0} \cong\left(\frac{\left(V_{\exp }\right)_{0}}{R_{0}}\right)\left(d_{g}\right)_{0} \cong H_{0}\left(d_{g}\right)_{0}
$$

When $\left(d_{g}\right)_{0} \rightarrow R_{0},\left(v_{g}\right)_{0} \cong H_{0} R_{0}$. This can be compared with currently believed Hubble's law for the current expanding universe.

\section{Results and Discussion}

\subsection{Cosmological constant problem}

With reference to proposed concepts, ratio of the Planck scale critical density to the current critical density is,

$$
\left(\frac{3 H_{p l}^{2} c^{2}}{8 \pi G}\right) \div\left(\frac{3 H_{0}^{2} c^{2}}{8 \pi G}\right) \cong\left(\frac{H_{p l}}{H_{0}}\right)^{2} \cong 6.685 \times 10^{121}
$$

We wish to appeal that, this idea can be considered as a characteristic tool for constructing a model of 'quantum gravity' with cosmic evolution.

\subsection{Horizon problem}

The 'horizon problem' is a problem with the standard cosmological model of the Big Bang. It points out that different regions of the universe have not 'contacted' each other because of the large distances between them, but nevertheless they have the same temperature and other physical properties. If one is willing to consider the concept of 'matter causes the space-time to curve', 'horizon problem' can be understood. According to hot big bang model, during its evolution, as the universe is expanding, thermal radiation temperature decreases and matter content increases. As 
matter content increases, based on Mach's principle, at any stage of evolution, it is possible to have an increasing radius of curvature, $R_{t} \cong \frac{G}{c^{2}}\left[\left(M_{O M}\right)_{t}+\left(M_{D M}\right)_{t}\right]$. For the current case, $R_{0} \cong \frac{G}{c^{2}}\left[\left(M_{O M}\right)_{0}+\left(M_{D M}\right)_{0}\right] \cong 10.40 \mathrm{Gpc}$ and there is no scope for 'causal disconnection' of distant visible matter.

\subsection{Cosmic inflation}

Basically, 'inflation' $[68,69]$ is a period of super cooled cosmic expansion, where temperature seems to be dropped by a factor of 100,000 and is mostly model dependent. Mainstream cosmologists believe that just after the hot big bang, 'inflation' seems to be started at $10^{-37}$ seconds and ended by $10^{-32}$ seconds. During this time span, cosmic temperature seems to be dropped from $10^{27} \mathrm{~K}$ to $10^{22}$ K. Since that time, expansion initially decelerated and then, after about 6 billion years, began very slowly to accelerate. Most puzzling point to be noted is that, the detailed mechanism responsible for inflation is still unknown. Many cosmologists proposed different starting mechanisms for initiating and fine tuning the believed 'inflation'. Since its introduction by Alan Guth in 1980, the inflationary paradigm has become widely accepted. Currently, a great debate is going on its existence [70,71]. In this proposed model, when $H_{t} \approx \frac{1}{t} \approx 10^{37} \mathrm{sec}^{-1}$, estimated $\left(\gamma_{t} \cong 15.43, T_{t} \cong 1.73 \times 10^{28} \mathrm{~K}\right)$. Similarly, when $H_{t} \approx \frac{1}{t} \approx 10^{32} \mathrm{sec}^{-1}$, estimated $\left(\gamma_{t} \cong 26.946, T_{t} \cong 4.14 \times 10^{25} \mathrm{~K}\right)$. Thus, for the currently believed inflationary time span of $\left(10^{-37}\right.$ to $\left.10^{-32}\right) \mathrm{sec}$, estimated ratio of initial and final temperatures seems to be around 400 and needs further study with respect to the believed ratio of 100000 . We are working in this direction.

\subsection{Cosmic acceleration and expansion velocity}

Before 1990s, most of the cosmologists thought that the cosmic expansion would be decelerating due to the gravitational attraction of the matter in the universe. In between 1995 and 1998, as space technology was advancing, based on the available supernovae redshift data, cosmologists noticed that, rather than slowing down, universe was expanding with increasing rate [72,73]. Even though it was a shocking news for most of the science community, confirmatory evidence has been found in baryon acoustic oscillations and other cosmological observations [74]. Considering the updated supernovae redshift data, in 2016, cosmologists noticed that, universe is coasting at constant speed rather than acceleration [Neilsen]. In this context, now a days, a great debate has been initiated among various groups of cosmologists on 'cosmic acceleration' $[16,75,76,77,78]$.

Now a days, main stream cosmologists are seriously working on 'eternal light speed expansion' [79, $80,81]$. In this context, in our earlier published papers, based on ordinary matter density and Hubble's law, we come across different magnitudes of cosmic expansion velocities ranging from $2 c$ to $12 c$. We would like to suggest that, by considering a decreasing trend of ordinary matter and dark matter density, starting from the Planck scale, it is possible to get an expression for cosmic expansion velocity comparable to speed of light. It can be expressed as follows.

$$
\frac{\left(V_{\text {exp }}\right)_{t}}{c} \cong \sqrt{\frac{2}{\left[\left(\Omega_{O M}\right)_{t}+\left(\Omega_{D M}\right)_{t}\right]}}
$$


Based on this expression, for the Planck scale, $\left(V_{\text {exp }}\right)_{p l} \cong c$ and for the current scale, $\left(V_{\text {exp }}\right)_{0} \cong 2.427 c$. Interesting point to be noted is that, after 20 billion years of cosmic expansion, increment in expansion velocity seems to be only $\left[\left(V_{\text {exp }}\right)_{0}-\left(V_{\text {exp }}\right)_{p l}\right] \cong 1.427 c$. See figure-3.

From figure 3, it is very clear that, right from the beginning of cosmic evolution, cosmic expansion velocity seems have an increasing trend. To some extent, this can be compared with currently believed cosmic acceleration concept. Interesting point to be noted is that, expansion velocity seems to depend on $\sqrt{\frac{2}{\left[\left(\Omega_{O M}\right)_{t}+\left(\Omega_{D M}\right)_{t}\right]}}$.

Figure 3: Increasing trend of cosmic expansion velocity

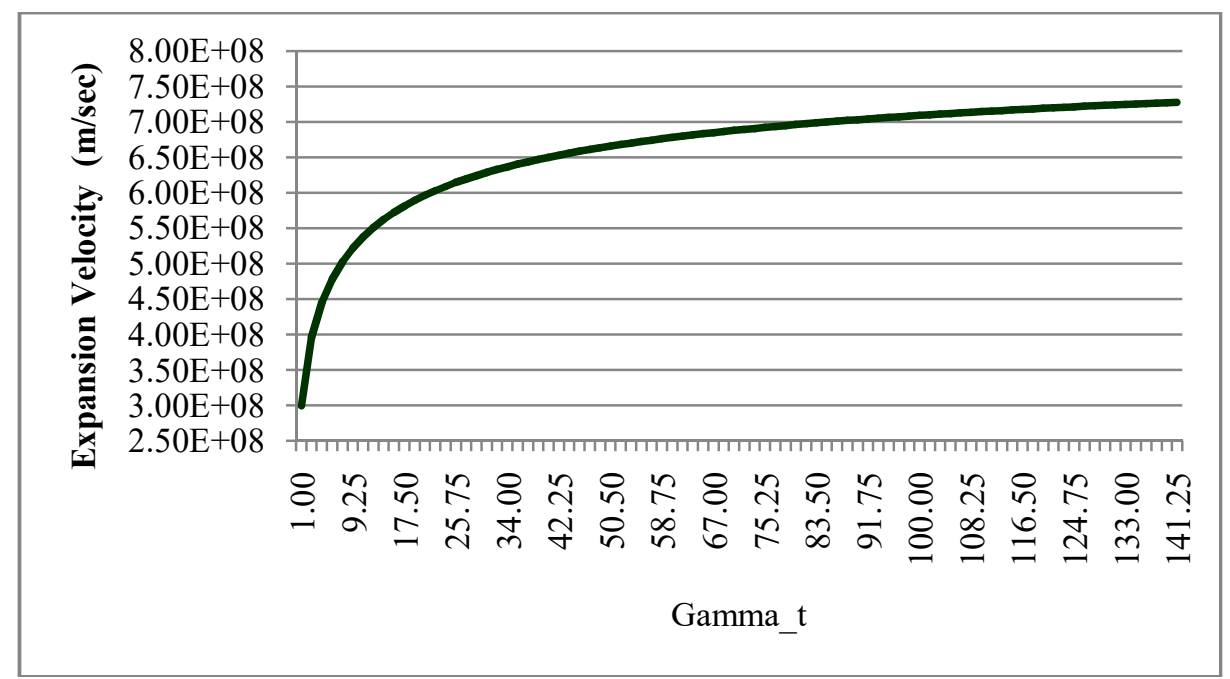

In near future, if decrease in $\left[\left(\Omega_{O M}\right)_{t}+\left(\Omega_{D M}\right)_{t}\right]$ is found to be significant, one can expect 'acceleration' and if decrease in $\left[\left(\Omega_{O M}\right)_{t}+\left(\Omega_{D M}\right)_{t}\right]$ is found to be insignificant, one can expect cosmic 'constant rate of expansion'. It is for further study.

\subsection{Cosmic angular velocity}

According to Haking S.W [43] - By vorticity one means the rotation of 'nearby' matter about an observer moving with the matter, relative to an inertial frame defined by gyroscopes. (Here 'nearby' should be interpreted as meaning at distances of about a hundred megaparsecs, to be near compared to the Hubble radius but far compared to the length scales of local phenomena such as the rotation of the Galaxy.) Thus in a sense, the whole Universe would be rotating, though, as the model is homogeneous, there would be no centre of rotation. Such rotation, if it existed, would be of great interest for the dynamical effects it could have on the Universe and on the formation of galaxies and for its relation to Mach's Principle. This states that the local inertial frame should be non-rotating with respect to distant matter.

With reference to our assumptions and relations, current angular velocity seems to be $\omega_{0} \cong 1.606 \times 10^{-20} \mathrm{rad} / \mathrm{sec} \cong 5.068 \times 10^{-13} \mathrm{rad} /$ year. This can be compared with other estimates. The first experimental evidence of the Universe rotation was done by Birch (1982), evidently. According to Birch, there appears to be strong evidence that the Universe is anisotropic on a large scale, producing position angle offsets in the polarization and brightness distributions of radio sources. These can 
probably be explained on the basis of a rotation of the Universe with an angular velocity of approximately $10^{-13} \mathrm{rad} /$ year. Now a days L.M. Chechin $[59,60]$ is seriously working on cosmic rotation.

According to Chechin [60], "In the early Universe, the orientation of its spin is random and the cosmological principle is satisfied. This result is naturally consistent with the CMB isotropy. In the modern Universe the rotation axis direction becomes anisotropic and the cosmological principle, strictly speaking, is not satisfied. This is confirmed by the large-scale anisotropy in the distribution of space objects and by the torque alignment direction. But since the value of the angular velocity of our Universe is $\omega_{U n} \sim 10^{-19} \mathrm{~Hz}$, finding of such rotation and its influence on the natural processes is extremely difficult. So today dominates the view that the Universe is isotropic and the cosmological principle is satisfied in it".

According to Godlowski [56], "Unfortunately, there is no relatively simple model of rotating universe derived from the general relativity theory, involving observables that could be used for its empirical testing. However, we can make some estimates of global rotation of the Universe on the grounds of the Newtonian equivalent of general relativity, while being fully aware that the Newtonian cosmology does not allow for describing actual evolution of the Universe, being but its conveniently applicable approximation. The situation is somewhat complicated by the fact that observationally it is very hard to distinguish the effects of global rotation from other effects, as the Casimir effect or dark radiation in the brane scenario of Randall-Sundrum. One can still estimate from the observational data the net contribution of all effects of this kind. It is shown that if it has a non-zero value, then one can explain the discrepancies between the values of matter density $\left(\Omega_{m}\right)_{0}$ and of the Hubble constant derived from measurements of SNIa and CMBR. It allows also to account for the discrepancies between the observed and theoretical abundances of helium-4 and deuterium".

According to Li-Xin Li [48],

1) Global rotation provides a natural origin for the rotation of galaxies, and the morphology of the objects formed from gravitational instability in a rotating and expanding universe depends on the amplitude of the density fluctuation, different values of the amplitude of the fluctuation lead to the formation of elliptical galaxies, spiral galaxies, and walls. The global rotation gives a natural explanation of the empirical relation between the angular momentum and mass of galaxies: $J \propto M^{\frac{5}{3}}$.

2) By studying the correlation between the angular momentum and mass of galaxies, it is possible to find the angular velocity of the universe.

3) Current derived cosmic angular velocity is $\omega_{0} \approx 6 \times 10^{-21} \mathrm{rad} / \mathrm{se} \approx 2 \times 10^{-13} \mathrm{rad} /$ year.

According to Yuri N. Obukhov [50],

1) However, "whether our universe is rotating or not, it is of fundamental interest to understand the interrelation between rotation and other aspects of cosmological models as well as to understand the observational significance of an overall rotation".

2) The possibility of combining cosmic rotation with expansion was the first successful step towards a realistic cosmology.

\section{Conclusions}


Planck mass can be considered as a characteristic representation of the mass of baby universe and Cosmological principle and big bang concepts can possibly be reviewed at fundamental level. Proceeding further, universe can be considered as a quantum gravitational object. Mach's relation can be given some consideration in developing a workable model of cosmology.

Even though it is very interesting, it is also true that the proposed relation $\gamma_{t} \cong\left(\frac{H_{t}}{\omega_{t}}\right) \cong\left[1+\ln \left(\frac{H_{p l}}{H_{t}}\right)\right] \cong \sqrt{\frac{3 H_{t}^{2} c^{2}}{8 \pi G\left(a T_{t}^{4}\right)}}$ is new and peculiar. We are confident that, its role, scope and validity can be verified with further study. Estimated current cosmic Planck ball radius is about 10.40 $\mathrm{Gpc}$ and can be compared with current observations of $14 \mathrm{Gpc}$. Current cosmic sphere seems to constitute around 14 Hubble spheres and needs further study with respect to the Bayesian model average estimate of $>251$ Hubble spheres proposed by Vardanyan et al [82].

We are working on understanding the scope, physical significance and validity of the proposed ad-hoc coefficients, $\beta_{t} \cong\left[\left(1+\sqrt{\gamma_{t}}\right) / 2\right]$ and $\beta_{t}^{2} \cong\left[\left(1+\sqrt{\gamma_{t}}\right) / 2\right]^{2}$. Trend of total matter density ratio seems to decide the fate of cosmic expansion rate and currently believed 'dark energy' concept can possibly be reviewed with respect to its real existence. Independent of galactic redshift data, we are working on finding alternative tools for understanding the cosmic expansion rate. In future, with advanced science, engineering and technology, by considering $\frac{d\left(T_{0}\right)}{d t}$ or $\frac{d\left(H_{0}\right)}{d t}$ or $\frac{d\left(\Omega_{O M}+\Omega_{D M}\right)_{0}}{d t}$, absolute cosmic rate of expansion can be estimated.

\section{Acknowledgements}

Author Seshavatharam is indebted to professors brahmashri M. Nagaphani Sarma, Chairman, shri K.V. Krishna Murthy, founder Chairman, Institute of Scientific Research in Vedas (I-SERVE), Hyderabad, India and Shri K.V.R.S. Murthy, former scientist IICT (CSIR), Govt. of India, Director, Research and Development, I-SERVE, for their valuable guidance and great support in developing this subject.

\section{References}

[1] Gamow G Nature 162 p 680 (1948)

[2] Gamow G Phys Rev 74 p 505 (1948)

[3] Abhas Mitra MNRAS 442 p 382 (2014)

[4] Abhas Mitra Journal of Modern Physics 2 p 1436 (2011)

[5] Annila A Mon.Not.Roy.Astron.Soc.423 p 1973 (2012)

[6] Grahn P, Annila A, Kolehmainen E AIP Advances 80350282018

[7] Arbab AI Spacetime \& Substance Vol 2 p 55 (2001)

[8] Arbab AI Gen.Rel. Grav 36 p 2465 (2004)

[9] Miyazaki A arXiv:gr-qc/0101.112v1 (2001)

[10] Bojowald M Rep. Prog. Phys 78 p 023901 (2015)

[11] Padmanabhan T Current Science 88 p 1057 (2005)

[12] Sivaram C Current Science Vol 79 p 25 (2000)

[13] Einstein A Ann. Phys. 360 p 241 (1918)

[14] Rennan Barkana Nature 555 p 71 (2018)

[15] J T Nielsen, A Guffanti and S Sarkar Scientific Reports 6 p 35596 (2016)

[16] Lawrence H Dam, Asta Heinesen and David L Wiltshire Mon.Not.Roy.Astron.Soc. 472 p 835 (2017) 
[17] J Colin, R Mohayaee, M Rameez and S Sarkar arXiv:1808.04597v1 (2018)

[18] Balakrishna S Haridasu, Vladimir V Lukovic, Rocco D’Agostino and Nicola Vittorio $A \& A \mathbf{6 0 0}$ L1 (2017)

[19] G'abor R'acz, L'aszl'o Dobos, R'obert Beck, Istv'an Szapudi and Istv'an Csabai Mon. Not. R. Astron. Soc. Lett. 469 L1 (2017)

[20] Lasha Berezhiani, Justin Khoury and Junpu Wang Phys. Rev. D 95 p 123530 (2017)

[21] Joel Smoller, Blake Temple and Zeke Vogler Proc. R. Soc. A 473201608872017

[22] Gong-Bo Zhao et al Nature Astronomy 1(9) p 627 (2017)

[23] Deng Wang and Xin-He Meng Phys. Rev. D 96 p 103516 (2017)

[24] Kate Land and Joao Magueijo Phys.Rev.Lett. 95 p 071301 (2005)

[25] Hutsem'ekers D Astron. Astrophys. 332 p 410 (1998)

[26] D Hutsemekers, R Cabanac, H Lamy and D. Sluse Astron. Astrophys. 441 p 15 (2005)

[27] Shamik Ghosh, Pankaj Jain, Gopal Kashyap, Rahul Kothari, Sharvari Nadkarni-Ghosh and Prabhakar Tiwari J. Astrophys. Astr. 37 p 25 (2016)

[28] Jain P Ralston J P Modern. Phys. Lett. A 14 p 417 (1999)

[29] Blake C Wall J Nature 416 p 150 (2002)

[30] Singal A K Astrophys.J. 742 L23 (2011)

[31] Gibelyou C and Huterer D MNRAS 427 p 1994 (2012)

[32] Tiwari P and Jain P MNRAS 447 p 2658 (2015)

[33] Rubart M and Schwarz D J Astrophys. Astr. 555 A p 1172013

[34] Tiwari P and Jain P MNRAS 460 (3) p 2698 (2015)

[35] Aluri P K and Jain P Modern. Phys. Lett. A 27(04) p 1250014 (2012)

[36] Rath P K, Mudholkar T, Jain P and Aluri P K JCAP 1304 p 007 (2013)

[37] Wen Zhao and Larissa Santos The Universe No 3 p 9 (2015)

[38] Whittaker ET Yearbook of Roy. Soc. Edinburgh 5 (1945)

[39] Gamow G Nature 158 No 4016 p 549 (1946)

[40] Godel K Rev. Mod. Phys. 21 p 447 (1949)

[41] Raychaudhuri A K Phys. Rev. 98 p 1123 (1955)

[42] Narlikar J V Mon. Not. R. Astr. Soc. 126 p 203 (1963)

[43] Hawking SW Mon. Not. R. Astr. Soc.142 p 129 (1969)

[44] Birch P Nature Vol 298 p 451 (1982)

[45] Korotky VA and Obukhov YuN JETP 81(6) p 103 (1995)

[46] Nodland B and Ralston J P. Phys. Rev. Lett. 78 p 3043 (1997)

[47] Rainer W K"uhne Mod. Phys.Lett. A 12 p 2473 (1997)

[48] Li-Xin Li General Relativity and Gravitation $30 \mathrm{p} 497$ (1998)

[49] Shwetabh Singh J. Astrophys. Astr. 20 p 67 (1999)

[50] Obukhov Y N Colloquium on Cosmic Rotation, Eds M. Scherfner, T. Chrobok and M. Shefaat (Wissenschaft und Technik Verlag: Berlin) p 23 (2000)

[51] Saulo Carneiro General Relativity and Gravitation Vol 34 p 793 (2002)

[52] Panov VF and Kuvshinova EV Gravitation \& Cosmology Vol 10 p 156 (2004)

[53] Barrow J D and Tsagas C G Class. Quantum. Grav. 21 p 1773 (2004)

[54] Godlowski W and Szydlowski Marek Gen.Rel.Grav. 37 p 907 (2005)

[55] Shi Chun Su and M-C Chu Ap.J 703 p 354 (2009)

[56] Godlowski W International Journal of Modern Physics D 20 p 1643 (2011)

[57] Longo M Phys. Lett. B 699 p 224 (2011)

[58] Rong-Gen Cai and Zhong-Liang Tuo JCAP 1202 p 004 (2012)

[59] Chechin LM Astron.Rep. 60 p 535 (2016)

[60] Chechin LM Gravit. Cosmol. 23 p 305 (2017)

[61] Tatum ET Seshavatharam U V S and Lakshminarayana S Journal of Applied Physical Science International $4 \mathrm{p} 18$ (2015) 
[62] Seshavatharam U V S and Lakshminarayana S Physical Science International Journal 14(2) p 1 (2017)

[63] Seshavatharam U V S and Lakshminarayana S Physical Science International Journal 15(4): 1 2017

[64] Seshavatharam U V S and Lakshminarayana S International Journal of Astronomy Astrophysics and Space Science 5(1) p 1 (2018)

[65] Seshavatharam U V S \& Lakshminarayana S Prespacetime journal Vol 9 p 326 (2018)

[66] Planck Collaboration: Planck 2015 Results XIII Cosmological Parameters (2015)

[67] Riess AG et al Astrophys.J 826 p 56 (2016)

[68] Guth AH Phys. Rev. D 23 p 347 (1981)

[69] Linde A Physics Letters B 108 p 389 (1982)

[70] Steinhardt PJ Scientific American 304 p 18 (2011)

[71] A Steinhardt P J and Abraham Loeb Physics Letters B 736 p 142 (2014)

[72] Riess AG et al Astron.J 116 p 1009 (1998)

[73] Perlmutter S et al Astrophys.J 517 p 565 (1999)

[74] Weinberg DH et al Physics Reports 530 p 87 (2013)

[75] Zhengxiang Li, Puxun Wu and Hongwei Yu Phys. Lett B 695 p 1 (2011)

[76] D Rubin and B Hayden The Astrophysical Journal Letters 833 L30 (2016)

[77] I Tutusaus, B Lamine and A Blanchard arXiv:1803.06197 (2018)

[78] Christos G Tsagas J. Phys: Conf. Ser. 283 p 012040 (2011)

[79] John MV Mon.Not.Roy.Astron.Soc. 000 p 1 (2016)

[80] Jun-Jie, Wei Xue-Feng Wu, Fulvio Melia and Robert S Maier The Astronomical Journal $149 \mathrm{p}$ $102(2015)$

[81] Fulvio Melia and Marco Fatuzzo Mon.Not.Roy.Astron.Soc. 456 p 3422 (2016)

[82] Mihran Vardanyan, Roberto Trotta and Joseph Silk MNRAS Lett 413 L91 (2011) 